\title{
Investigation of the Stiffness and Strength of Particulate Composites by Means of a Variant Cubic Model and SEM Fractography Microscopic Approach
}

\author{
Emilio Sideridis, Victor Kytopoulos, Antonis Kampouroglou, John Venetis ${ }^{*}$ \\ School of Applied Mathematical and Physical Sciences, National Technical University of Athens, Greece
}

Copyright $\subset 2018$ by authors, all rights reserved. Authors agree that this article remains permanently open access under the terms of the Creative Commons Attribution License 4.0 International License

\begin{abstract}
In this article the stiffness of particulate composites is determined by the use of a multivariant three-phase model. This model consists of the transformation of spatial cubic models; it simulates a particulate composite into a three-sphere model, designating the two main phases of the composite material, the filler and the matrix, and applying the classical theory of elasticity to it. Theoretical results derived from this model are compared with experimental results derived from tensile tests carried out with iron particle reinforced epoxy resin composites and also with other theoretical results given by other researchers. In this context, an attempt is made to give, in a somewhat tentative way, a semiquantitative explanation of certain discrepancies observed between experimental data and the theory concerning the elastic modulus as well as experimental data concerning some fracture parameters on the basis of a macroscopic and a fractography-aided microscopic approach.
\end{abstract}

Keywords Particulate Composites, Particle Contiguity, Variable Modulus Interphase, Stiffness

\section{Introduction}

Composite materials are among the materials that have recently undergone a marked degree of technological development and their use has increased in recent years. Their complete characterization has not been achieved so far; this is due to complex material properties such as chemical compatibility, wettability, absorption characteristics, and stress development owing to differences in expansion. The addition of metal particles into polymeric matrices results in composite materials that are characterized by enhanced mechanical properties such as their moduli and their fracture toughness.

However, one of the main problems remains the prediction of the composite properties when the properties of the constituent materials are known. This problem arises from the fact that the thermomechanical properties of a composite depend on a large number of parameters, such as the individual filler and the matrix, the particle size and size distribution, the filler volume fraction, the quality of adhesion between filler and matrix, the mode of filler packing, etc. A number of theoretical analyses, which define the mechanical properties of composites and give equations for predicting the thermoelastic constants, have been reported in the literature, emphasizing particular parameters [1-21]. The filler concentration and the mode of packing were the parameters studied in Refs. [1-3] whereas the influence of the particle size on the final properties of the composites was discussed in Refs. [4-6], [12-13]. Christensen and Lo [7] and Christensen [8] derived a three-phase model, the generalized self-consistent model, which is an improved version of Kerner's model. The effect of the shape of the inclusions was investigated by Chow [9] who applied successfully Eshelby's solution. Finally the influence of the filler-matrix adhesion on the mechanical behavior of composites has been discussed in a series of models presented in Refs. [10], [11], [13], [15], [17-21]. Meanwhile, the effect of various parameters on the thermal properties of particulate composites was studied in [22-26]. In the past years, there have been many research works carried out for the determination of the properties of particulate composites and for the investigation of the effect of filler-matrix, particle interaction. In Ref. [27], accurate approximate relations for the effective elastic moduli of composite materials were obtained, whereas in [28], a new approach for bounding the effective moduli was developed. Besides, the effective elastic constants of solids containing random arrangements of spherical inclusions were considered in [29]. Meanwhile, Yin and Sun [30] investigated the mechanical properties of periodic composites containing identical spherical particles using the principles of micromechanics. According to them, the 
particle interactions make no contribution to the effective modulus.

In this article, the stiffness of a particle-reinforced composite material was studied. Variations of a microstructural composite model of a regular geometry and topology simulating the basic cell of the composite were adopted. This model was transformed to a three-phase spherical representative volume element in order to apply the classical theory of elasticity for a solution to the problem. The theoretical results derived from this multivariant model were compared with experimental results carried out with iron particle reinforced epoxy resin and also with other theoretical values obtained from expressions existing in the literature, for strength [31-33] and for stiffness $[2,35-40]$.

\section{Theoretical Analysis}

\section{The Proposed Model}

As is known, microstructural models aim at the reproduction of the basic cell or representative volume elements of the composite at a macroscopic scale in order that a solution be obtained. These models are usually based on the following assumptions: A regular geometric form is adopted for the inclusions, usually a sphere or cylinder. On special occasions, other forms are adopted as well, such as needle- or ribbon-like inclusions or sharp-angled forms. Regular geometry and topology are adopted for the model. Experimental models can be plane or spatial. It is obvious that a three-dimensional structure is synonymous with a composite material structure. However, the assumptions of plane stress and plane strain may provide useful information by much simpler means on many occasions. The model adopted is presented in Fig. 1a-I-III. It represents a three-dimensional system capable of simulating real particle composites. Assuming equal inclusions, which is not necessarily the case in reality, the volume fraction, Uf, of the fillers in the continuous phase, the matrix, is given in terms of the ratio $2 r_{f} / l$ where $r_{f}$ is the filler radius and $l$ the intercenter distance respectively. We assume that the inclusions occupy the eight corners (vertices) of a cube (see Fig. 1a-I), that they are placed at the middle of each one of the twelve sides of the cube (see Fig. 1a-II), or finally, that they are placed at the center of each one of the six faces (see Fig. 1a-III).

Thus, we have the models designated as M1, M2, and M3 following assumptions, given that the volume fraction, $U_{f}$, and the radius, $r_{f}$, of the inclusions are known.
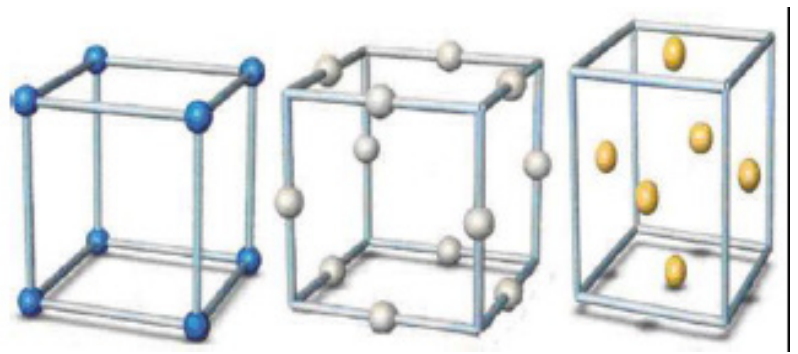

Figure 1a. Typical cubic models: (i) simple cubic model; (ii) side centered cubic model;(iii) face centered cubic model.

A cube of side $2 l$ surrounding the cube of side $l$ constitutes the unit cell with it (see Fig. 1b). And this array continues in all materials. Thus, we have the unit cells of the models designated as M1, M2, and M3 respectively.

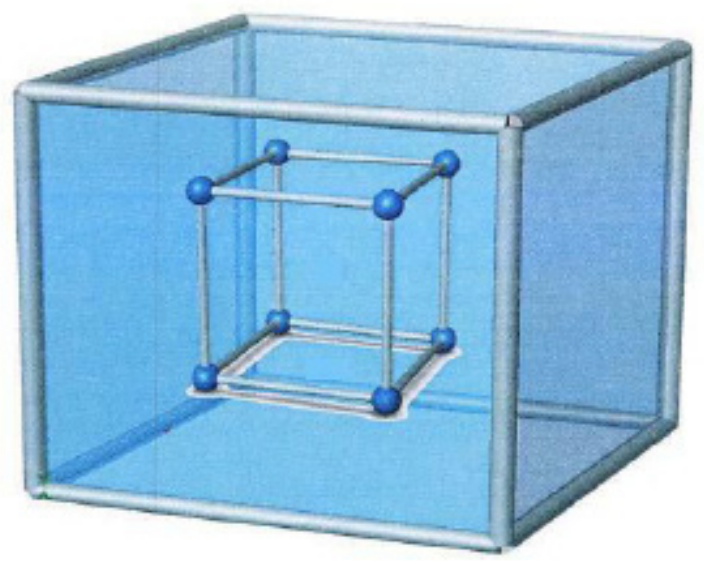

Figure 1b. Unit cell of the simple cubic model

In order that the analysis be easier, we may transform the above cubic volume element into a 3-phase spherical model as it can be seen in Fig. 1c

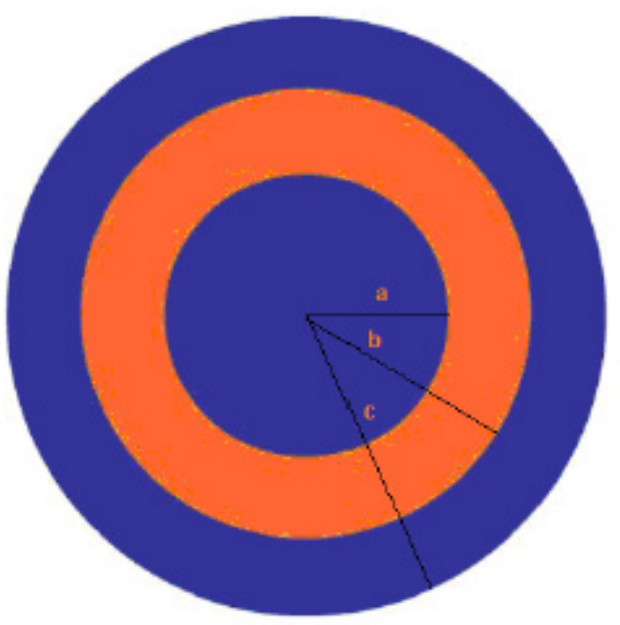

Figure 1c. Three Phase model 
A sphere with radius $r_{m, 2}=c$ having equal volume with the cube of side $2 l$.

A concentric sphere with radius $r_{m, l}=\alpha$, which simulates the matrix in the cube of side $l$.

A concentric hollow sphere of radii $r_{m, l}=a$ and $r_{f}=b$, which simulates the neighboring inclusions of the cube of side 1 and whose volume is equal to the volume of these inclusions.

A concentric hollow sphere of radii $r_{f}=b$ and $r_{m, 2}=c$, which simulates the matrix in the cube of side $2 l$.

Next we should determine the unknown radii with respect to the filler radius $r_{f}$ and volume fraction $U_{f}$ which are the parameters that characterize the given particulate composite.

We can write the following relationships in order to calculate the radii $a, b$, and $c$. The total volume fraction for the three different cubic models is given as follows

$$
U_{f}=\frac{8\left(\frac{4}{3} \pi r_{f}^{3}\right)}{2 l^{3}}
$$

or

$$
U_{f}=\frac{12\left(\frac{4}{3} \pi r_{f}^{3}\right)}{2 l^{3}}
$$

or

$$
U_{f}=\frac{6\left(\frac{4}{3} \pi r_{f}^{3}\right)}{2 l^{3}}
$$

The volume of the cube with side $2 l$ is equal to the volume of the sphere with radius $c$ :

$$
(2 l)^{3}=\frac{4}{3} \pi c^{3} \rightarrow c=l^{3} \sqrt{\frac{6}{\pi}}
$$

which, together with Eqs. 1a-c, yields

$$
c=r_{\mathrm{f}} \sqrt[3]{\frac{8}{v_{\mathrm{f}}}}
$$

or

$$
c=r_{\mathrm{f}}^{8} \sqrt{\frac{12}{v_{\mathrm{f}}}}
$$

or

$$
c=r_{t}^{3} \sqrt{\frac{6}{v_{f}}}
$$

for M1, M2, and M3 respectively.

Now, let us consider the cube with side $l$. The length of the main diagonal of this cube is $l \sqrt{3}$, whereas the diagonal of each face the cubc is $l \sqrt{2}$. If we denote by $w$ the distance from the center of the cube to one vertex or to the middle of a side or to the center of a face (see Fig. 1a), we have

$$
w=l \frac{\sqrt{3}}{2}
$$

or

$$
w=l \frac{\sqrt{2}}{2}
$$

or

$$
w=\frac{l}{2}
$$

for variations $\mathrm{M} 1, \mathrm{M} 2$, and $\mathrm{M} 3$ respectively again.

We consider that the volume of the hollow spherical region with radii $a$ and $b$ is distributed in equal parts of volume on both sides of the spherical surface of radius $w$. Thus,

$$
\frac{4}{3} \pi\left(b^{3}-w^{3}\right)=\frac{4}{3} \pi\left(w^{3}-a^{3}\right) \rightarrow a^{3}+b^{3}=w^{3}
$$

The volume, however, of the second region is equal to the volume of the eight, twelve, or six spherical inclusions depending on each one of the adopted variations Ml, M2, and M3 of the model.

Thus,

$$
\frac{4}{3} \pi\left(b^{3}-a^{3}\right)=12\left(\frac{-4}{3} \pi r_{f}^{3}\right) \rightarrow b^{3}-a^{3}=8 r_{f}^{3}
$$

or

$$
\begin{aligned}
& \frac{4}{3} \pi\left(b^{3}-a^{3}\right)=12\left(-\pi r_{f}^{3}\right) \rightarrow b^{3}-a^{3}=12 r_{f}^{3} \\
& \text { or } \\
& \frac{4}{3} \pi\left(b^{3}-a^{3}\right)=12\left(-\pi r_{f}^{3}\right) \rightarrow b^{3}-a^{3}=12 r_{f}^{3}
\end{aligned}
$$

The solution of Eqs. and $5 a-c$ yields

$$
a=\sqrt[3]{w^{3}-4 r_{\mathrm{f}}^{3}} \text { and } b=\sqrt[3]{w+4 r_{\mathrm{f}}^{3}}
$$

or

$$
a=\sqrt[3]{w^{3}-6 r_{f}^{3}} \text { and } b=\sqrt[3]{w+6 r_{f}^{3}}
$$

or

$$
a=\sqrt[3]{w^{3}-4 r_{f}^{3}} \text { and } b=\sqrt[3]{w+4 r_{f}^{3}}
$$

for M1, M2, and M3 respectively. Since c $>\mathrm{b}>\mathrm{a}$, using Eqs. $1-5$ we obtain

$$
U_{r}<\frac{\pi}{3}\left(\frac{\sqrt{3}}{2}\right)^{3} \rightarrow U_{\mathrm{f}}<0.68
$$

or

$$
U_{r}<\frac{\pi}{3}\left(\frac{\sqrt{2}}{2}\right)^{3} \rightarrow U_{\mathrm{f}}<0.37
$$


or

$$
U_{\mathrm{f}}<\frac{\pi}{3}\left(\frac{1}{2}\right)^{3} \rightarrow U_{\mathrm{f}}<0.13
$$

for variations M1, M2, and M3 respectively again. These values constitute the limit values of the filler volume fraction.

\section{Theoretical Considerations}

The theoretical analysis for the determination of the elastic modulus is based on the following assumptions:

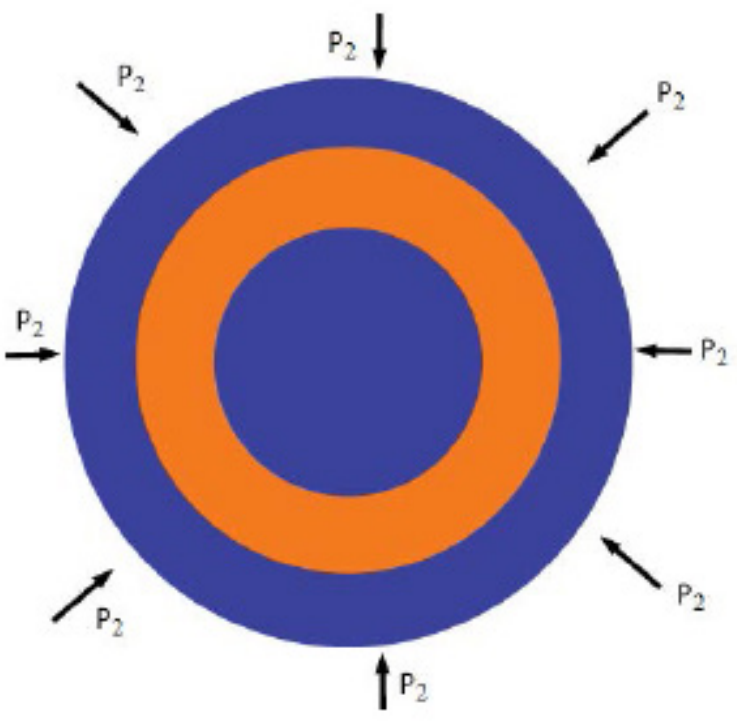

Figure 2. Schematic representation of the composite stresses

(i) The matrix and the particles are elastic, isotropic, and homogeneous.

(ii) The particles are perfectly spherical in shape.

(iii) The particles are large in number, and their distribution is uniform so that the composite may be regarded as a quasi-homogeneous isotropic material.

(iv) The deformations applied to the composite are small enough to maintain linearity of stress-strain relations.

The expression for the elastic modulus of the composite can be obtained by applying the classical theory of elasticity to the representative volume element whose mechanical properties equal to the average properties of the composite and which can be represented by the previously described three concentric spheres. Let a pressure $P_{2}$ be applied on the outer surface of the sphere with radius $c$. Then $P_{l}$ denotes the interaction (common stress) between filler and matrix, $P_{0}$ the interaction between filler and remaining matrix region (See Fig. 2).

Because of the spherical symmetry, it is convenient to use spherical coordinates $(r, \theta, \varphi)$. Only $u_{r}$ is non zero of the three components of the displacement vector $u_{r}, u_{\theta}, u_{\varphi}$.

The solution to this problem can be approached by a stress function of the following form:

$$
\Phi=\frac{C_{1}}{r}+C_{2} r^{2}
$$

Where $C_{1}$ and $C_{2}$ are constants.

Each one of the three regions has its own stress function:

$$
\Phi_{1}=\frac{A}{r}+B r^{2}, \Phi_{2}=\frac{C}{r}+D r^{2}, \Phi_{3}=\frac{H}{r}+F r^{2}
$$

To avoid infinite stresses at $r=0$, the constant $\mathrm{A}$ must be equal to 0 , i.e. $\mathrm{A}=0$.

Thus, $\Phi_{1}=B r^{2}$

The displacements can be obtained from the stress functions through the relationship

$$
\bar{u}=\frac{1}{2 G} \operatorname{grad} \Phi
$$

Then the displacements are given as

$$
\begin{aligned}
& u_{r_{1}}=\frac{B r}{G_{1}} \\
& u_{r_{r}}=\frac{-\frac{C}{r^{2}}+2 D r}{2 G_{2}} \\
& u_{r 3}=\frac{-\frac{H}{r^{2}}+2 F r}{2 G_{3}}
\end{aligned}
$$

where $G$ denotes the shear modulus, given that $u_{\theta}=u_{\phi}$ $=0$. Here, the indices 1 and 3 designate the two matrix regions whereas the index 2 the equivalent filler region.

The strains for each region can be determined from the relationships

$$
\varepsilon_{r}=\frac{\partial u_{r}}{\partial r}, \quad \varepsilon_{\theta}=\frac{u_{r}}{r}+\frac{1 \partial u_{\theta}}{r \partial \theta}, \quad \varepsilon_{\phi}=\frac{u_{r}}{r}+\frac{1 \partial u_{\phi}}{r \partial \phi}
$$

Taking into consideration that $u_{\phi}=u_{\theta}=0$ and the relationship

$$
G_{i}=\frac{E_{i}}{\left(1+v_{i}\right.}
$$

where $E_{i}$ is the elastic modulus and $v_{\mathrm{i}}$ the Poisson's ratio of each phase

$$
\begin{gathered}
\varepsilon_{r, 1}=\varepsilon_{\theta 1}=\varepsilon_{\varphi, 1}=B \frac{1+v_{1}}{E_{1}} \\
\varepsilon_{r, 2}=\left(\frac{2 C}{r^{3}}+2 D\right) \frac{1+v_{2}}{E_{2}} \\
\varepsilon_{\theta, 2}=\varepsilon_{\phi, 2}=\left(-\frac{C}{r^{8}}+2 D\right) \frac{1+v_{2}}{E_{2}} \\
\varepsilon_{r, 3}=\left(\frac{2 H}{3}+2 F\right) \frac{1+v_{s}}{E_{g}}
\end{gathered}
$$




$$
\varepsilon_{\theta, 3}=\varepsilon_{\phi, 3}=\left(-\frac{H}{r^{3}}+2 F\right) \frac{1+v_{8}}{E_{8}}
$$

The stresses for each region can be found from the stress-strain relationships

$$
\begin{gathered}
\sigma_{r, 1}=\sigma_{\theta 1}=\sigma_{\varphi 1}=\frac{2\left(1+v_{1}\right)}{1-2 v_{2}} B \\
\sigma_{r, 2}=\frac{2 C}{r^{8}}+\frac{2\left(1+v_{2}\right)}{1-2 v_{2}} D \\
\sigma_{\theta, 2}=\sigma_{\phi, 2}=-\frac{c}{r^{8}}+\frac{2\left(1+v_{2}\right)}{1-2 v_{2}} D \\
\sigma_{r, 3}=\frac{2 H}{r^{3}}+\frac{2\left(1+v_{3}\right)}{1-2 v_{3}} F \\
\sigma_{\theta, 3}=\sigma_{\phi 3}=-\frac{H}{r^{8}}+\frac{2\left(1+v_{8}\right)}{1-2 v_{3}} F
\end{gathered}
$$

The boundary conditions for the stresses are as follows :

$$
\begin{array}{cr}
r=a: & \sigma_{r, 1}=\sigma_{r, 2}=-P_{0} \\
\text { At } r=b: & \sigma_{r 2}=\sigma_{r 3}=-P_{1} \\
r=c: & \sigma_{r, 3}=-P_{2}
\end{array}
$$

Equations 16-20 applied to the above boundary conditions yield the values of the constants:

$$
\begin{gathered}
B=-\frac{P_{0}\left(1-2 v_{1}\right)}{2\left(1+v_{1}\right.} \\
C=\frac{\left(P_{1}-P_{0}\right) a^{3} b^{8}}{2\left(b^{3}-a^{8}\right)} \\
D=\frac{\left(a^{8} P_{0}-b^{8} P_{1}\right)\left(1-2 v_{2}\right)}{2\left(b^{8}-a^{3}\right)\left(1+v_{2}\right.} \\
H=\frac{\left(P_{2}-P_{1}\right) b^{8} c^{3}}{2\left(c^{3}-b^{8}\right)} \\
F=\frac{\left(b^{8} P_{1}-c^{8} P_{2}\right)\left(1-2 v_{8}\right)}{2\left(c^{8}-a^{8}\right)\left(1+v_{3}\right.}
\end{gathered}
$$

By substituting these values in Eqs. 8-10

$$
\begin{gathered}
u_{r_{1}}=-\frac{P_{0}\left(1-2 v_{1}\right)}{E_{1}} r \\
u_{r, 2}=-\frac{\left(P_{1}-P_{0}\right) a^{8} b^{8}\left(1+v_{2}\right)}{2\left(b^{3}-a^{8}\right) E_{2}} \frac{1}{r^{2}}+\frac{\left(a^{3} P_{0}-b^{8} P_{1}\right)\left(1-2 v_{2}\right)}{\left(b^{8}-a^{8}\right) E_{2}} r \\
U_{r, 3}=-\frac{\left(P_{2}-P_{1}\right) b^{8} c^{8}\left(1+v_{3}\right)}{2\left(c^{8}-b^{8}\right) E_{3}} \frac{1}{r^{2}}+\frac{\left(b^{8} P_{1}-c^{8} P_{1}\right)\left(1-2 v_{3}\right)}{\left(c^{3}-b^{8}\right) E_{3}} r
\end{gathered}
$$

The boundary conditions must account for the continuity of displacements at the interfaces and they are expressed as follows:

$$
\text { At } \begin{aligned}
r & =a: & u_{r, 1}=u_{r, 2} \\
r & =b: & u_{r, 2}=u_{r, 3}
\end{aligned}
$$

From Eqs. $25-27$ at $\mathrm{r}=\alpha$ we obtain

$$
\begin{aligned}
& -\frac{P_{0}\left(1-2 v_{1}\right)}{E_{1}} a=-\frac{\left(P_{1}-P_{0}\right) a^{3} b^{3}\left(1+v_{2}\right)}{2\left(b^{3}-a^{5}\right) E_{2}} \frac{1}{a^{2}}+\frac{\left(a^{3} P_{0}-b^{3} P_{1}\right)\left(1-2 v_{2}\right)}{\left(b^{3}-a^{3}\right) E_{2}} a \\
& \Rightarrow P_{1}\left[3 b^{3}\left(1-v_{2}\right) E_{1}\right]=P_{0}\left\{2\left(b^{3}-a^{3}\right)\left(1-2 v_{1}\right) E_{2}+\right. \\
& \left.\left[b^{3}\left(1+v_{2}\right)+2 a^{3}\left(1-2 v_{2}\right)\right] E_{1}\right\} \Rightarrow \\
& P_{0}=\frac{3 b^{3}\left(1-v_{2}\right) E_{1}}{\left\{2\left(b^{3}-a^{3}\right)\left(1-2 v_{1}\right) E_{2}+\left[b^{3}\left(1+v_{2}\right)+2 a^{3}\left(1-2 v_{2}\right)\right] E_{1}\right\}} P_{1}
\end{aligned}
$$

By setting

$\mu=\frac{\left[3 b^{8}\left(1-v_{2}\right) E_{1}\right]}{\left\{2\left(b^{8}-\alpha^{8}\right)\left(1-2 v_{1}\right) E_{2}+\left[b^{8}\left(1+v_{2}\right)+2 \alpha^{8}\left(1-2 v_{2}\right)\right] E_{1}\right\}}$

Eq 29 becomes

$$
P_{0}=\mu P_{1}
$$

From Eqs. 27 and 28 at $r=b$ we obtain

$$
\begin{aligned}
& -\frac{\left(P_{1}-P_{0}\right) a^{3} b^{3}\left(1+v_{2}\right)}{\left(b^{3}-a^{3}\right) E_{2}} \frac{1}{b^{2}}+\frac{\left(a^{3} P_{0}-b^{3} P_{1}\right)\left(1-2 v_{2}\right)}{\left(b^{3}-a^{3}\right) E_{2}} b \\
= & -\frac{\left(P_{2}-P_{1}\right) c^{3} b^{3}\left(1+v_{3}\right)}{\left(c^{3}-b^{3}\right) E_{2}} \frac{1}{b^{2}}+\frac{\left(b^{3} P_{1}-c^{3} P_{1}\right)\left(1-2 v_{3}\right)}{\left(c^{3}-b^{3}\right) E_{3}} b \\
\Rightarrow & P_{0}\left[3 a^{3}\left(1-v_{2}\right)\left(c^{3}-b^{3}\right) E_{3}\right]+P_{2}\left[3 c^{3}\left(1-v_{3}\right)\left(b^{3}-a^{3}\right) E_{2}\right] \\
= & P_{1}\left\{\left[a^{3}\left(1+v_{2}\right\}+2 b^{3}\left(1-2 v_{2}\right)\right]\left(c^{3}-b^{3}\right) E_{3}+\left[c^{3}\left(1+v_{3}\right)+\right.\right. \\
& \left.\left.2 b^{3}\left(1-2 v_{3}\right)\right]\left(b^{3}-c^{3}\right) E_{2}\right\}
\end{aligned}
$$

By substituting in $E q .31$ the expression for $P_{0}$ from $E q$. 30, we obtain

$$
\begin{gathered}
P_{2}\left[3 c^{3}\left(1-v_{3}\right)\left(b^{3}-a^{3}\right) E_{2}\right]= \\
=P_{1}\left\{\left[a^{3}\left(1+v_{2}\right\}+2 b^{3}\left(1-2 v_{2}\right]\left(c^{3}-b^{3}\right) E_{3}+\left[c^{3}\left(1+v_{3}\right)+\right.\right.\right. \\
\left.\left.\left.b^{3}\left(1-2 v_{3}\right)\right]\left(b^{3}-c^{3}\right) E_{2}-3 \mu a^{3}\left(1-v_{2}\right)\right]\left(c^{3}-b^{3}\right) E_{3}\right\}
\end{gathered}
$$

By setting

$\lambda=\frac{3 c^{3}\left(1-v_{3}\right)\left(b^{3}-a^{3}\right) E_{2}}{\left.\left\{\left[a^{3}\left(1+v_{2}\right\}+2 b^{3}\left(1-2 v_{2}\right)\right]\left(c^{3}-b^{3}\right) E_{3}+\left[c^{3}\left(1+v_{3}\right)+2 b^{3}\left(1-2 v_{3}\right)\right]\left(b^{3}-c^{3}\right) E_{2}-3 \mu a^{3}\left(1-v_{2}\right)\right]\left(c^{3}-b^{3}\right) E_{3}\right\}}$

Eq. 32 becomes

$$
P_{1}=\lambda P_{2}
$$

From Eqs 30 and 33

$P_{0}=\mu \lambda P_{2}$, and denoting $: \kappa=\mu \lambda$ we have

$$
P_{0}=\kappa P_{2}
$$

Next we substitute the obtained values of the constants (Eqs. 21-25) in Eqs. 16-20 for the stresses and in Eqs. 
11-15 for the strains and we obtain

$$
\begin{gathered}
\sigma_{r, 1}=\sigma_{\theta, 1}=\sigma_{\phi 1}=-P_{0} \\
\sigma_{r, 2}=\frac{\left(P_{1}-P_{0}\right) a^{8} b^{3}}{\left(b^{8}-a^{8}\right)} \frac{1}{r^{3}}+\frac{a^{8} P_{0}-b^{8} P_{1}}{b^{3}-a^{3}} \\
\sigma_{\theta, 2}=\sigma_{\phi, 2}=-\frac{\left(P_{1}-P_{0}\right) a^{8} b^{8}}{2\left(b^{8}-a^{3}\right)} \frac{1}{r^{3}}+a^{3} P_{0}-b^{3} P_{1} b^{3}-a^{3} \\
\sigma_{r, 3}=\frac{\left(P_{2}-P_{1}\right) b^{3} c^{8}}{\left(c^{8}-b^{8}\right)} \frac{1}{r^{8}}+\frac{b^{3} P_{1}-c^{8} P_{2}}{c^{7}-b^{8}} \\
\sigma_{\theta 3}=\sigma_{\phi, 3}=-\frac{\left(P_{2}-P_{1}\right) b^{8} c^{8}}{2\left(c^{8}-b^{8}\right)} \frac{1}{r^{8}}+\frac{b^{3} P_{1}-c^{3} P_{2}}{c^{8}-b^{3}}
\end{gathered}
$$

And

$$
\begin{gathered}
\varepsilon_{r, 1}=\varepsilon_{\theta, 1}=e_{\phi 1}=-\frac{P_{0}\left(1-2 v_{1}\right)}{E_{1}} \\
\varepsilon_{r, 2}=\frac{\left(P_{1}-P_{0}\right) a^{8} b^{8}\left(1+v_{2}\right)}{\left(b^{8}-a^{8}\right) E_{2}} \frac{1}{r^{8}}+\frac{\left(1-2 v_{2}\right)\left(a^{8} P_{0}-b^{8} P_{1}\right)}{E_{2}}
\end{gathered}
$$

$\varepsilon_{\theta, 2}=\varepsilon_{\phi, 2}=-\frac{\left(P_{1}-P_{0}\right) a^{8} b^{3}\left(1+v_{2}\right)}{2\left(b^{8}-a^{3}\right) E_{2}} \frac{1}{r^{8}}+\frac{\left(1-2 v_{2}\right)}{E_{2}} \frac{\left(a^{8} P_{0}-b^{3} F\right.}{\left(b^{8}-a^{3}\right)}$

$$
\varepsilon_{r, 3}=\frac{\left(P_{2}-P_{1}\right) b^{8} c^{8}\left(1+v_{3}\right)}{\left(c^{8}-b^{3}\right) E_{3}} \frac{1}{r^{3}}+\frac{\left(1-2 V_{2}\right)}{E_{3}} \frac{\left(b^{8} P_{1}-c^{8} P_{2}\right)}{c^{3}-b^{3}}
$$

$$
\varepsilon_{\theta, 3}=\varepsilon_{\phi, 3}=-\frac{\left(P_{2}-P_{1}\right) b^{3} c^{8}\left(1+v_{8}\right)}{2\left(c^{8}-b^{8}\right) E_{3}} \frac{1}{r^{8}}+\frac{\left(1-2 v_{g}\right)}{E_{8}} \frac{\left(b^{3} P_{1}-c^{8}\right.}{c^{8}-b^{3}}
$$

The elastic modulus of the composite can be obtained by applying the energy balance to the spherical composite model. The strain energy of the composite must be equal to the sum of the strain energies of the three regions (phases):

$$
\begin{aligned}
\frac{1}{2} \int_{c} \frac{P_{2}^{2}}{K_{c}} d V_{c} & =\frac{1}{2} \int_{1}\left(\sigma_{r 1} \varepsilon_{r, 1}+\sigma_{\theta, 1} \varepsilon_{\theta, 1}+\sigma_{\phi, 1} \varepsilon_{\phi, 1}\right) d V_{1} \\
& +\frac{1}{2} \int_{2}\left(\sigma_{r, 2} \varepsilon_{r, 2}+\sigma_{\theta, 2} \varepsilon_{\theta, 2}+\sigma_{\phi, 2} \varepsilon_{\phi, 2}\right) d V_{2} \\
& +\frac{1}{2} \int_{3}\left(\sigma_{r, 3} \varepsilon_{r, 3}+\sigma_{\theta, 3} \varepsilon_{\theta, 3}+\sigma_{\phi, 3} \varepsilon_{\varphi, 3}\right) d V_{3}
\end{aligned}
$$

where

$$
K_{c}=\frac{E_{C}}{3\left(1-2 \mathbf{v}_{c}\right)}
$$

is the bulk modulus of the composite and $d V=4 \pi r^{2} \mathrm{dr}$. By substituting Eqs. 35-39 for the stresses and Eqs. 40-44 for the strains, after some algebra, we obtain the following relationship:

$$
\begin{gathered}
\frac{P_{2}^{2}\left(1-2 v_{c}\right) c^{3}}{E_{c}}=\frac{P_{0}^{2}\left(1-v_{1}\right) a^{3}}{E_{1}}+\frac{\left(P_{1}-P_{0}\right)^{2} a^{3} b^{3}\left(1+v_{1}\right)}{\left(b^{3}-a^{3} E_{2}\right.} \\
+\frac{\left(1-2 v_{2}\right)}{E_{2}} \frac{\left(a^{3} P_{0}-b^{3} P_{1}\right)^{2}}{b^{3}-a^{3}}+\frac{\left(P_{2}-P_{1}\right) 2 b^{3} c^{3}\left(1+v_{3}\right)}{\left(c^{3}-b^{3} E_{3}\right.} \\
+\frac{\left(1-2 v_{3}\right)}{E_{3}} \frac{\left(b^{3} P_{1}-c^{3} P_{2}\right)^{2}}{c^{3}-b^{3}}
\end{gathered}
$$

The volume fractions of the three regions according to the considered model are given as

$$
U_{m 1}=U_{1}=\frac{V_{1}}{v_{c}}=\frac{\frac{4}{3} \pi a^{3}}{\frac{3}{3} \pi c^{3}}=\frac{a^{3}}{c^{3}}
$$

$$
\begin{gathered}
U_{f}=U_{2}=\frac{V_{2}}{V_{c}}=\frac{\frac{4}{3} \pi b^{3}-\frac{4}{3} \pi a^{3}}{\frac{4 \pi c^{3}}{3}}=\frac{b^{3}-a^{3}}{c^{3}} \\
U_{m 2}=U_{3}=\frac{V_{3}}{V_{c}}=\frac{\frac{4}{3} \pi c^{3}-\frac{4}{8} \pi^{8}}{\frac{4}{3} \pi c^{8}}=\frac{c^{3}-b^{8}}{c^{8}}
\end{gathered}
$$

Taking into account Eqs. 33 and 34 from where $\lambda=\frac{P_{1}}{P_{2}}$ and $x=\frac{P_{0}}{P_{2}}, E q s .48-50$, and by dividing the two parts of $E q .47$ with $P_{2}^{2} c^{3}$, we find the following final relationship for $E_{c}$ :

$$
\begin{aligned}
& \frac{2\left(1-2 v_{c}\right)}{E_{*}}=2 \kappa^{2} U_{1} \frac{\left(1-2 v_{1}\right)}{E_{1}} \\
& +\frac{1}{E_{2}}\left\{\frac{U_{1}\left(1-U_{3}\right)(\lambda-\kappa)^{2}\left(1+v_{2}\right)}{U_{2}}+\frac{\left[U_{1} \kappa-\left(1-U_{3} \lambda\right)\right]^{2}\left(1-2 v_{2}\right)}{U_{2}}\right\} \\
& +\frac{1}{E_{3}}\left\{\frac{\left(1-U_{3}\right)(1-\lambda)^{2}\left(1+V_{3}\right)}{U_{3}}+\frac{2\left[\left(1-U_{3}\right) \lambda-1\right]^{2}\left(1-2 v_{3}\right.}{U_{3}}\right\}
\end{aligned}
$$

The Poisson's ratio, $v_{c}$ of the composite can be calculated by the inverse mixtures law or the mixtures law, since the difference between the Poisson's ratios of matrix and fillers is small. Thus,

$$
\frac{1}{v_{c}}=\frac{w_{1}}{v_{1}}+\frac{u_{2}}{v_{2}}+\frac{v_{3}}{v_{3}}
$$

or

$$
v_{c}=v_{1} U_{1}+v_{2} U_{2}+v_{3} U_{3}
$$

\section{Experimental Work}

Tensile tests with real composites were carried out in order to compare the experimental results obtained by these tests with the theoretical values derived by the proposed model and also with the theoretical values calculated by the aid of theoretical expressions given by other researchers.

The matrix material was a diglycidyl ether of bisphenolA resin, with the commercial designation Epikote 828 
(Shell), cured with $8 \mathrm{phr}$ triethylene tetramine hardener. It was filled with iron particles with average radius $r_{f}=150$ $\mu \mathrm{m}$ at volume fractions $U_{\mathrm{f}}=0.05,0.10,0.15,0.20$, and 0.25 . The elastic moduli of the matrix and the filler are 3.5 and $210 \mathrm{GPa}$ respectively whereas their Poisson's ratio are 0.36 and 0.29 respectively.

The procedure of preparing the mixture of the liquidmatrix phases and the iron particles is similar to that used in previous works of the researchers.

Dogbone specimens with a constant cross section at the measuring region of $\left(6 \times 10^{-3}\right) \times\left(3 \times 10^{-3}\right) \mathrm{m}^{2}$ and a length of $45 \times 10^{-3} \mathrm{~m}$ were used during the tests that were carried out with a Instron-type testing machine at room temperature. The specimens were tested at a rate of extension of $2 \times 10^{-3} \mathrm{~m} / \mathrm{min}$. To obtain the stress-strain diagrams, strain gauges were located on the specimens for the measurement of the strains.

Four point bending experiments were carried on prismatic specimens with dimensions of $190 \times 10^{-3} \mathrm{~m}$ and $10 \times 10^{-3} \mathrm{~m}$ of the same composite material according to ASTM D790 for $\mathrm{U}_{f}=0,0.10,0.15$ to evaluate the Poisson's ratio by using longitudinal and transversal strain gauges, in order to obtain the load-strain diagrams.

Concerning the SEM-aided investigations, the fractured surfaces of the specimens were coated with a conductive thin gold layer in a coating unit by means of DC-sputtering procedure. The applied gold layer avoids electron charging effects which tend to damage the material and degrade the photograph; however, at the same time it tends to improve the image quality by enhanced emission of secondary electrons. The fractographical data were taken by means of an S4-10 type scanning electron microscope from Cambridge Instruments.

\section{Results and Discussion}

\section{Macroscopic Approach}

Figure 3 presents the variation of stress versus the longitudinal strain for various filler contents $\left(\mathrm{U}_{\mathrm{f}}=0-0.25\right)$ of the iron particle reinforced epoxy polymer as obtained from tensile tests. We can observe that as $\mathrm{U}_{\mathrm{f}}$ increases, the linear part of the stress-strain diagram decreases. This can be attributed to the epoxy resin being viscoelastic whereas the iron particles being elastic material. It can be said that their addition in the epoxy polymer reinforces the elastic behavior of the composite.

In Figure 4 the variation of the tensile fracture stress, $\sigma_{\mathrm{c}}$, versus the filler content, as obtained from the experiments, is presented. It can be observed that $\sigma_{c}$, decreases as the filler content increases. This can be attributed to the shape of the fillers, the aspect ratio of which is small (nominally 1 ) and they create a reduction of the strength of the matrix acting, to some extent, as voids. Thus, the composite behaves as approximately an array of resin-bonded particles, in contrast to the case when reinforcing with inclusions with relatively high aspect ratio that ensure a load transfer through the matrix and increase its strength. In the same figure the theoretical values as derived from the equations of Nielsen [31], Nicolais-Narkis [32] and Shrager [33] given in the Appendix A are added. It can be observed that there is a good agreement between these values and the experimental ones.

\section{STRESS- STRAIN}

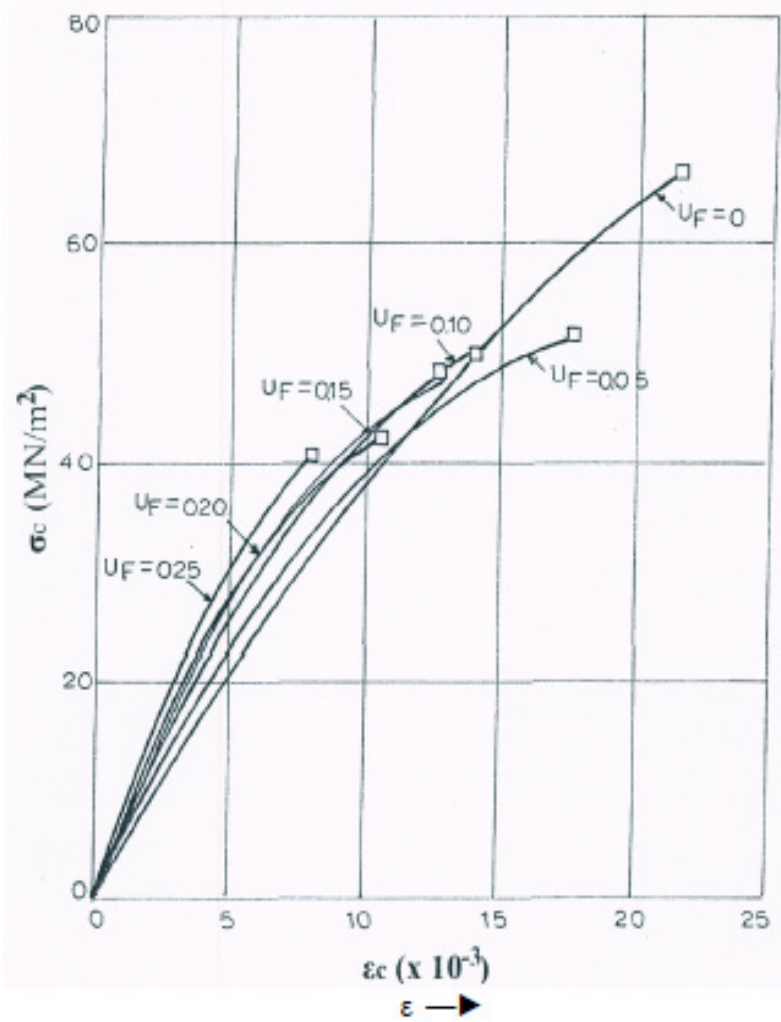

Figure 3. Stress-strain curves obtained from tensile experiments for different filler volume fractions

It is known that the stiffness and the strength of a composite are influenced by several factors, among them the interaction of the matrix and the inclusions. And this is because of the different structure of an intermediate phase, the interphase, developed around the inclusions, which is an important factor affecting the mechanical and physicochemical properties of the composite material. However, it can be said that usually the stiffness and the strength of the composite material vary with the content and the type of the inclusions. The theoretical limit of the filler content is determined by the quantity of the inclusions that makes probable the existence of a certain interphase or interlayer between inclusions and matrix [34]. Thus, the reinforcement of polymers by the addition of small particles is due to the formation of a continuous frame work, in a certain manner, as a result of the interaction of the particles.

Figure 5 presents the variation of Poisson's ratio, $\mathrm{v}_{\mathrm{c}}$, versus the filler content, $U_{f}$, as obtained from Eqs. 52a and 
52b. It can be observed that the Poisson's ratio decreases when $\mathrm{U}_{f}$ is increased. This means that the addition of iron particles in the epoxy polymer reduces not only the longitudinal strain, as observed in Figure 3, which is expected since the iron particles added are elastic, whereas the epoxy resin is viscoelastic and thus its ductility is reduced, but also the transversal strain, resulting in a decreased Poisson's ratio for the composite material. As it can be seen from Table 1, the values obtained from the two formulae are too close since the Poisson's ratios of the matrix and filler are also too close and thus the elastic modulus is not influenced much. Also, the comparison with the experimental data obtained from bending experiments for $U_{f}=0, U_{f}=0.10$, and $U_{f}=0.15$ shows a good agreement for these contents. Of course, more data are necessary in order to have a better idea. Unfortunately the existing data for this material are limited.

In Figure 6 the variation of the elastic modulus, $E_{c}$, versus the filler content is presented as derived from the three variations of the proposed theoretical model and other theoretical expressions mentioned in Appendix (Eqs. A1A8). It can be seen that all the models predict an increase in $E_{c}$ when increasing the particle volume fraction. Almost, all theoretical models present a similar variation except that of Guth-Smallwood (Eq. A3), which shows an abrupt increase vs. $U_{f}$. The experimental values as derived from tensile tests are also presented in the same figure. It can be seen that there is no coincidence between these values and those derived from the variations (M1) and (M3) of the proposed model and that the experimental results are, in all cases, too far from the theoretical ones. On the contrary, the theoretical values derived from the variation (M2) of this model are in good agreement with experimental results. Thus, it can be concluded that the distribution of particles influences the elastic modulus. Among the other theoretical models, those of Guth-Smallwood [35,36] and Paul [37] yield values that are too high when compared with experimental results. On the contrary, the values derived from Einstein [38] and Takahashi [39] are too low in comparison with experimental results. Moreover, the theoretical values derived from Kerner 2 model [2] and two-phase model are too close to the values obtained from variation (M3) of the proposed model. Finally, the Counto model [40] yields values that are too close to those derived from the variation (M2) of the proposed model and they are in good agreement with experimental results for $\mathrm{U}_{f}=0.05$ and 0.10 , whereas for higher values of filler content the values derived from variation M2 are in better agreement with the values derived from the experiments. All the theoretical and experimental values are presented in Table 2. A possible improvement would be the obtained from the consideration of the adhesion between the two main phases by means of a boundary interphase layer. Also, to carry out experiments with different inclusion diameters since it is known that stiffness and strength are influenced by this factor.

\section{FRACTURE STRESS}

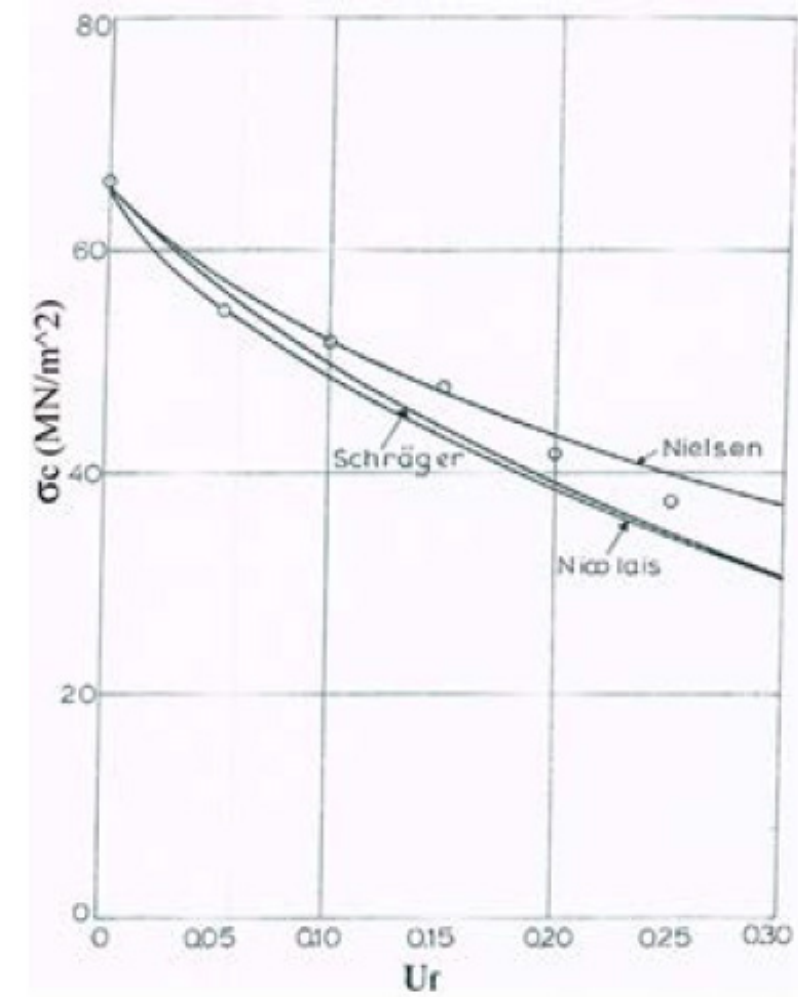

Figure 4. Variation of the tensile stress at fracture of the composite vs. filler content 


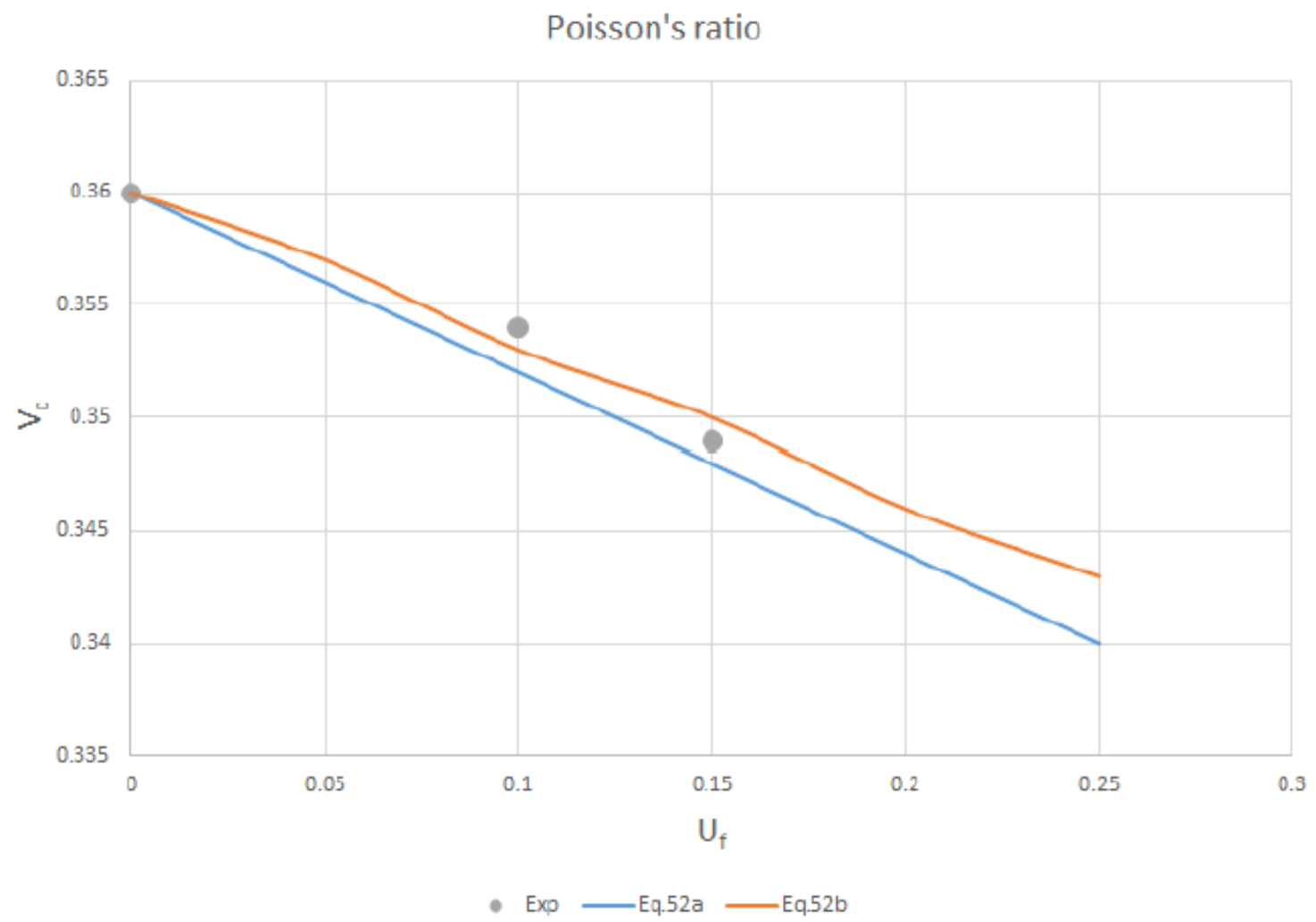

Filler volume fraction $0 \%$

Filler volume fraction $10 \%$.

Filler volume fraction $15 \%$
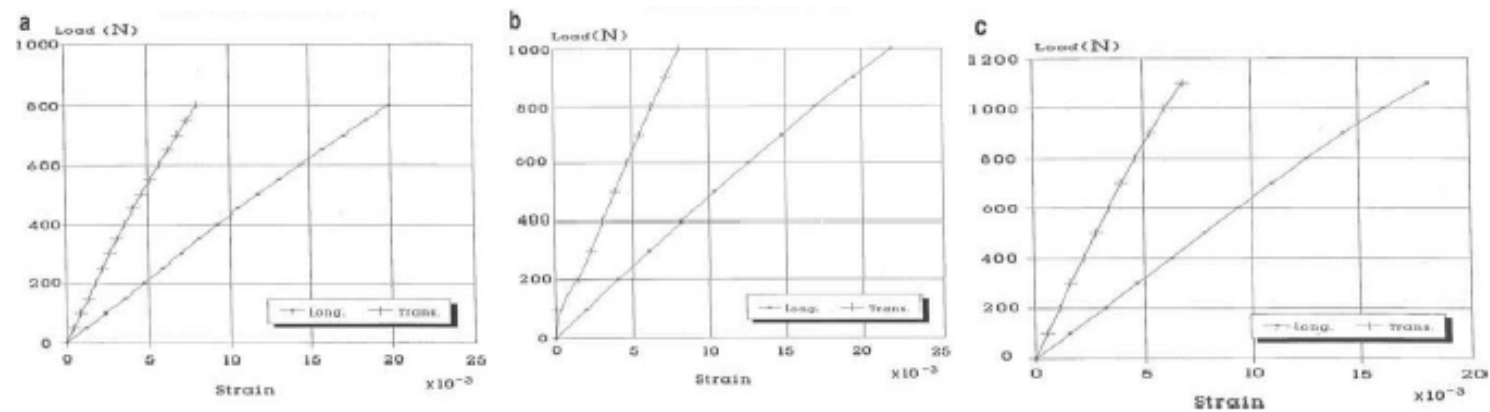

Figure 5. (a) Variation of the Poisson's ratio of the composite vs. filler content as derived by the proposed model, (b) Load-strain curves obtained from bending experiments for $\mathrm{Ur}=0,0.10$, and 0.15 .

Table 1. Theoretical results for Poisson's ratio.

Poisson's ratio $\left(\mathrm{v}_{\mathrm{c}}\right)$

\begin{tabular}{lccc}
\cline { 2 - 4 } $\begin{array}{c}\text { Filler content } \\
\left(\mathrm{U}_{\mathrm{f}}\right)\end{array}$ & $\begin{array}{c}\text { Equation } \\
52 a\end{array}$ & $\begin{array}{c}\text { Equation } \\
52 b\end{array}$ & $\begin{array}{c}\text { Experimental } \\
\text { values }\end{array}$ \\
\hline & 0.360 & 0.360 & 0.360 \\
0.05 & 0.356 & 0.357 & \\
0.1 & 0.352 & 0.353 & 0.354 \\
0.15 & 0.348 & 0.350 & 0.349 \\
0.2 & 0.344 & 0.346 & \\
0.25 & 0.340 & 0.343 & \\
\hline
\end{tabular}




\section{ELASTIC MODULUS}

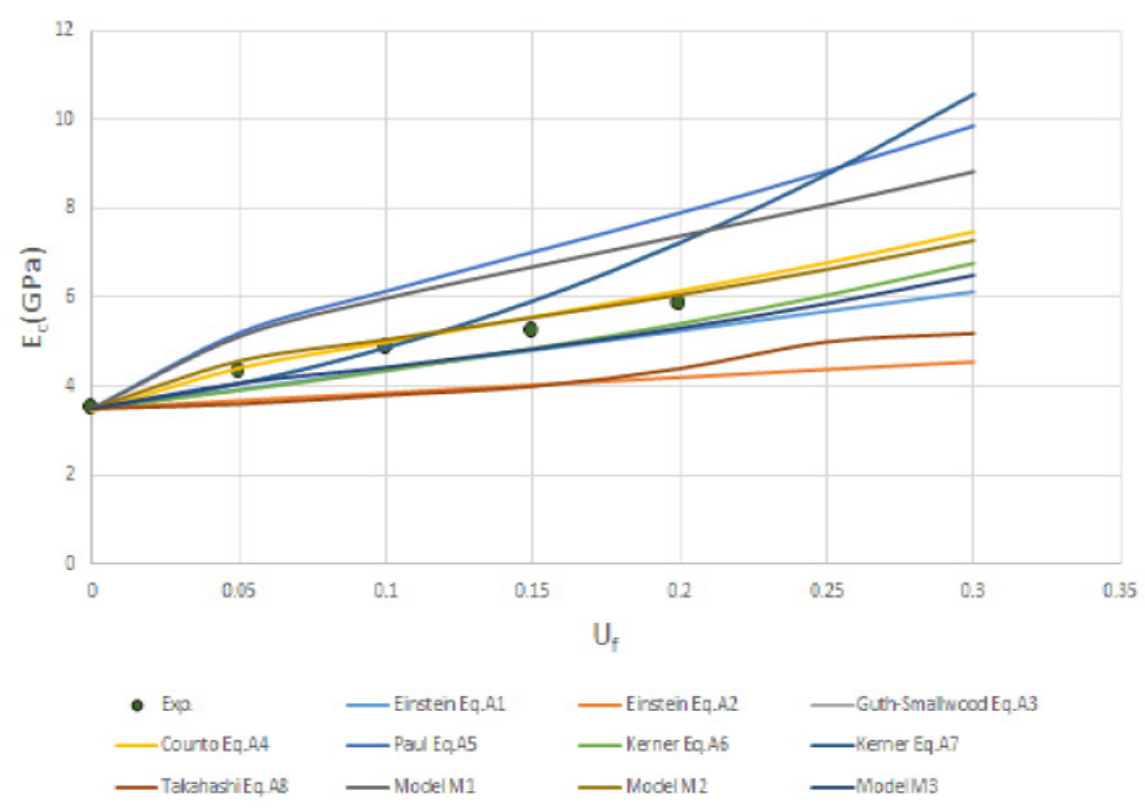

Figure 6. Variation of the elastic modulus of the composite vs. filler content as derived by the proposed model and other theoretical models. Comparison with experimental results

It can be deduced that the addition of iron particles in epoxy polymer influences in a different manner the strength and the stiffness of the created composite material. Its stiffness is usually greater than that of the matrix material whereas its strength is less. This can be better understood in terms of the "stiffness factor" and the "reinforcement factor", the first being the ratio of the composite modulus to that of the epoxy matrix and the second the ratio of the tensile fracture stress of the composite to that of the matrix material. As can be seen in Figure 7 , the stiffness factor is always greater than 1 whereas reinforcement factor is always less than 1 .

The reinforcement of the matrix material because of the addition of inclusions may be related to the work of fracture $\mathrm{W}$ of the polymer as it is evaluated from the area below the stress-strain curve:

$$
W=\int_{l_{0}}^{l_{\mathrm{f}}} \sigma d l
$$

where $l_{0}$ is the initial length of the specimen, $l_{f}$ its length at fracture, and $\sigma$ the applied stress. The augmentation of the work of fracture per unit volume, by the addition of particles, can be considered as a basic characteristic of the reinforcement action of the fillers in a polymer at the elastic state. The amount of the increase or decrease in the work of fracture depends on the nature of the filler.

The variation of the work of fracture per unit volume (toughness) versus filler content is presented in Figure 7. It can be seen that the relative fracture energy $\frac{W_{c}}{W_{m}}$, where $\mathrm{W}_{\mathrm{c}}$ and $W_{m}$ denote the fracture energy of the composite and the matrix respectively, decreases, at first abruptly then smoothly, when increasing the filler volume fraction. Thus, according to those previously mentioned the iron particles can be considered as inactive.

\section{Microscopic Approach}

From the inspection of Figure 6 concerning the modulus it can be deduced that none of the mentioned theoretical models can fit perfectly the experimental data. In other words, some of these models can respond only in part to the experimental values. This discrepancy was expected because most theoretical assumptions and conceptions cannot be fulfilled in praxis. Thus, for example, one of these conceptions considers filler particles as spheres of approximately uniform size with smooth surfaces. As can be seen from the electron fractographs (Fig. 8), this is not the case. Rather, the size and shape of the particles are not uniform and their surfaces are not smooth. Moreover, the latter have many crevices in which portions of macromolecules of the polymeric matrix may be trapped. This fact can lead to a nonuniform strain and stress field concentrations around the particles and thus to a strong nonhomogeneous localized behavior. Further, other conceptions consider a homogeneous distribution of the filler particles in the matrix. Again, from the fractographs (Fig. 8a-8c) it can be seen that this is not the case. Rather, a inhomogeneous particle distribution, such as agglomeration across the surface can be observed. This inhomogeneity in turn may lead to an enhancement of the above-mentioned nonuniformity of the local stress-strain fields between the particles. 
Table 2. Results for elastic modulus Elastic modulus $\mathrm{E}_{\mathrm{c}}(\mathrm{GPa})$

\begin{tabular}{|c|c|c|c|c|c|c|c|c|c|c|c|c|}
\hline \multirow{2}{*}{$\begin{array}{c}\text { Filler content } \\
U_{\mathrm{f}} \\
\end{array}$} & \multirow[b]{2}{*}{\begin{tabular}{|l} 
Einstein \\
$($ Eq.Al) \\
\end{tabular}} & \multirow[b]{2}{*}{\begin{tabular}{|l} 
Einstein \\
(Eq.A2) \\
\end{tabular}} & \multirow[b]{2}{*}{$\begin{array}{c}\text { Guth-Smallwood } \\
\text { (Eq. A3) }\end{array}$} & \multirow[b]{2}{*}{$\begin{array}{l}\text { Counto } \\
\text { (Eq.A4) }\end{array}$} & \multirow[b]{2}{*}{$\begin{array}{c}\text { Paul } \\
(E q . A 5)\end{array}$} & \multirow[b]{2}{*}{$\begin{array}{l}\text { Kerner } \\
\text { (Eq.A6) }\end{array}$} & \multirow[b]{2}{*}{$\begin{array}{l}\text { Kerner } \\
(\text { EqA7) }\end{array}$} & \multirow[b]{2}{*}{$\begin{array}{l}\text { Takahashi } \\
\text { (Eq.A8) }\end{array}$} & \multicolumn{3}{|c|}{ Present model (Eq. 5l) } & \multirow{2}{*}{$\begin{array}{c}\text { Experimental } \\
\text { results }\end{array}$} \\
\hline & & & & & & & & & $\begin{array}{c}\text { Model } \\
\text { Ml }\end{array}$ & $\begin{array}{c}\text { Model } \\
\text { M2 }\end{array}$ & $\begin{array}{c}\text { Model } \\
\text { M3 }\end{array}$ & \\
\hline 0 & 3.50 & 3.50 & 3.50 & 3.44 & 3.50 & 3.50 & 3.50 & 3.50 & 3.50 & 3.50 & 3.50 & 3.50 \\
\hline 0.05 & 3.93 & 3.68 & 4.06 & 4.41 & 5.20 & 3.91 & 4.06 & 3.60 & 5.12 & 4.57 & 4.07 & 4.34 \\
\hline 0.10 & 4.38 & 3.85 & 4.87 & 4.99 & 6.14 & 4.35 & 4.87 & 3.80 & 5.98 & 5.05 & 4.43 & 4.86 \\
\hline 0.15 & 4.81 & 4.03 & 5.92 & 5.56 & 7.02 & 4.85 & 5.92 & 4 & 6.69 & 5.55 & 4.84 & 5.24 \\
\hline 0.20 & 5.25 & 4.2 & 7.23 & 6,15 & 7.9 & 5.41 & 7.23 & 4.40 & 7.38 & 6.06 & 5.31 & 5.85 \\
\hline $\begin{array}{l}0.25 \\
0.30\end{array}$ & $\begin{array}{l}5.69 \\
6.13\end{array}$ & $\begin{array}{l}4.38 \\
4.55\end{array}$ & $\begin{array}{r}8.77 \\
10.57\end{array}$ & $\begin{array}{l}6.78 \\
7.48\end{array}$ & $\begin{array}{l}8.84 \\
9.86\end{array}$ & $\begin{array}{l}6.05 \\
6.77\end{array}$ & $\begin{array}{r}8.77 \\
10.57\end{array}$ & $\begin{array}{l}5 \\
5.19\end{array}$ & $\begin{array}{l}8.08 \\
8.83\end{array}$ & $\begin{array}{l}6.63 \\
7.28\end{array}$ & $\begin{array}{l}5.86 \\
6.50\end{array}$ & 6.45 \\
\hline
\end{tabular}

REL WORK OF FRACTURE (TOUGHNESS), REL STIFFNESS REINFORCEMENT FACTOR, REL FRACTURE STRAIN

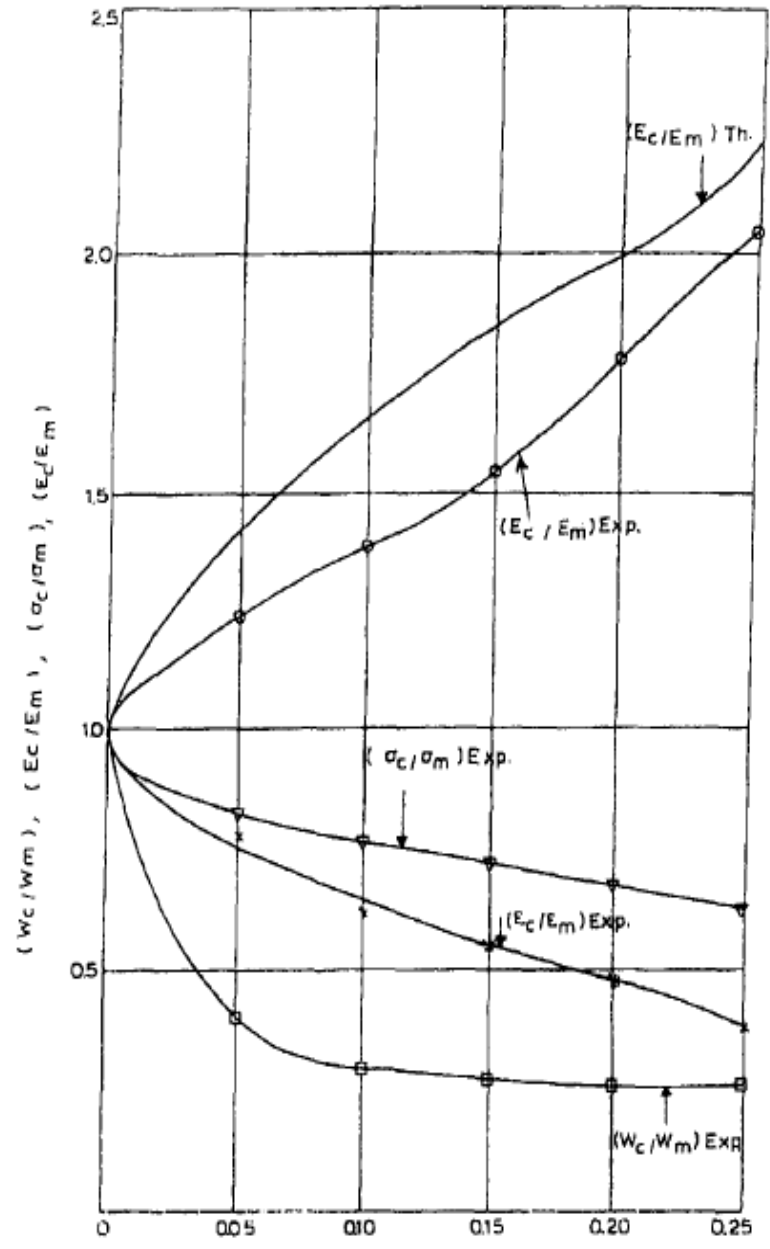

Figure 7. Variation of the stiffness factor, reinforcement factor, and the fracture energy of the composite vs. filler content

At this moment we can go a step further to explain the above mentioned discrepancies by the implementation of the previously mentioned interphase concept [41], According to this concept the composite may be considered as consisting of three phases: the matrix, the inclusion, and a third phase, as a boundary layer of variable thickness $(\Delta r)$. This layer is formed because of polymerization rate effects during setting of the matrix, as well as the mobility of the macromolecules in the contact zone. Thus, the local variation of the thickness $(\Delta r)$ of this layer affects the molecular bonding between matrix and inclusion and thus can lead to changes in the gross mechanical behavior of the composite concerning the elastic modulus and fracture. Regarding the elastic modulus, it can be shown that (see Appendix B)

$$
E \quad \frac{[A m(m-n]}{r_{0}^{m+1}}
$$

or

$$
E \propto \frac{1}{r_{0}^{m+1}}
$$

where $A, m$, and $n$ are constants and $\mathrm{r}_{\mathrm{n}}$ is the effective equilibrium interatomic (intermolecular) spacing.

This means, as it is shown in the following, that the modulus can locally be strongly influenced by small (finite) changes $(\Delta r)$ in the intermolecular spacing $r_{0}$. Even so, such small local variations or changes may be caused in the thickness of the interphase because of the previously mentioned nonuniform strain-stress field concentrations around individual inclusion. Furthermore, from the discrepancy between the theoretical and experimental fracture surface energy [42] as well as the appearance and analysis of the fracture surfaces obtained by electron fractography such as of Figure 8 , it can be concluded that the polymeric (matrix) material may respond to the local stress around the grains or at the tip of the internal flaw in a ductile rather than in a brittle manner, despite that, macroscopically, the behavior would be characterized as brittle. Such an "inherent" ductile response should involve a considerable amount of a highly localized segmental chain transport, which is sustained mainly by the changes in the van der Waal's secondary binding forces, rather than covalent primary bonds. From the above, it seems plausible to assume that the changes in the inter-phase thickness, $(\Delta \mathrm{r})$, must involve changes mainly in van der Waal's forces, and for such forces in $E q .54$ we can take $m=6$ and $n=11$ [43]. In this way and by the semiquantitative approach given in detail in Appendix B, we can estimate a measure for the earlier-discussed changes in the "local" modulus as $\frac{\Delta \tilde{E}}{\tilde{E}} \cong \pm 0.80$. This means that the maximal changes in the local modulus $\tilde{E}$, due to interphase thickness variations, $\pm \Delta \mathrm{r}$, can virtually be of the order of \pm $80 \%$, which may lead to appreciable discrepancy from the theoretical predictions concerning the gross (macroscopically) measured modulus, $E$. 
By means of the stress-strain curves given in Figure 3, we have constructed the curves given in Figure 7 relating the relative fracture stress and strain as well as the relative toughness behavior in function of the filler volume fraction Uf. From these plots the monotonic decrease of the first two parameters with a trend of an "asymptotical stabilization" of the relative toughness with in-creasing particle content can be observed. For instance, it can be plausible to explain this by observing the stress- strain

a

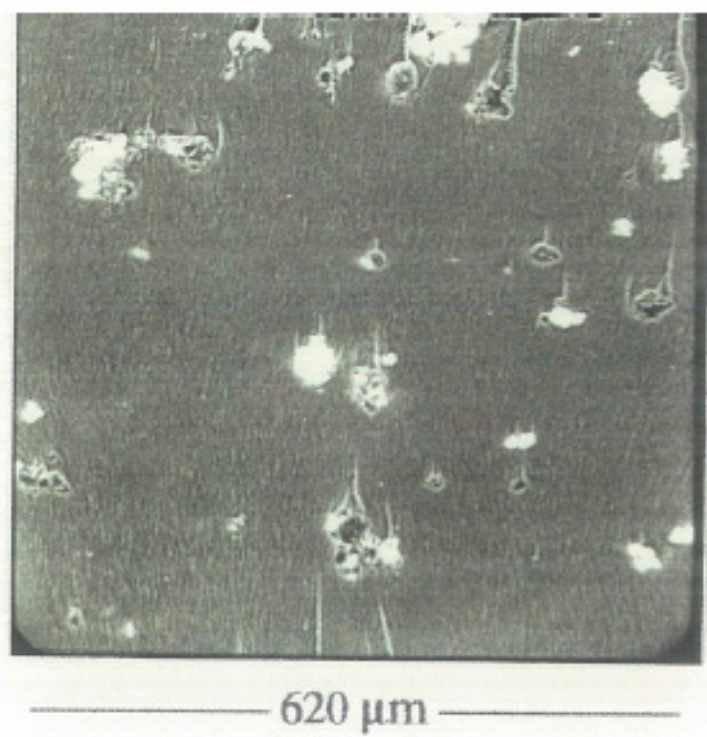

C

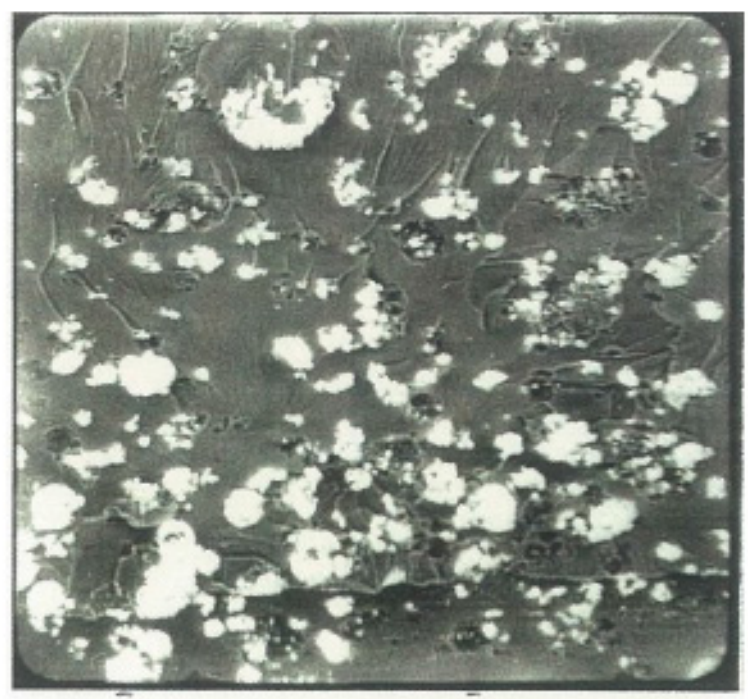

$620 \mu \mathrm{m}$ curves given in Figure 3: the decrease in the fracture stress and strain tends to be "compensated" by an increase in the "slope" of the curve and in this sense to contribute to the area under the curve that expresses the fracture energy per unit volume or the toughness of the material. But as it can be observed from the same figure (Fig. 3), the slope of each curve is not totally linear, Rather, there is a strong nonlinear behavior of each slope.

b

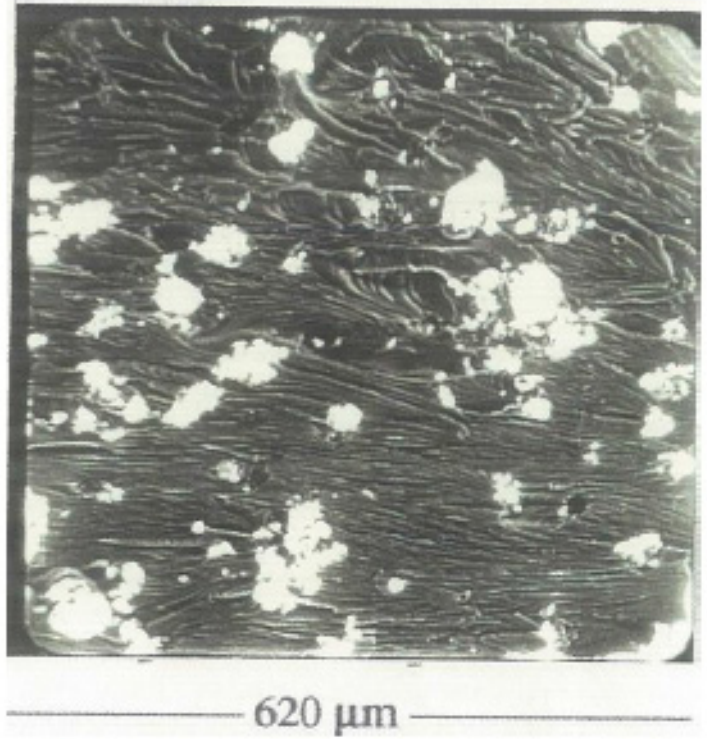

d

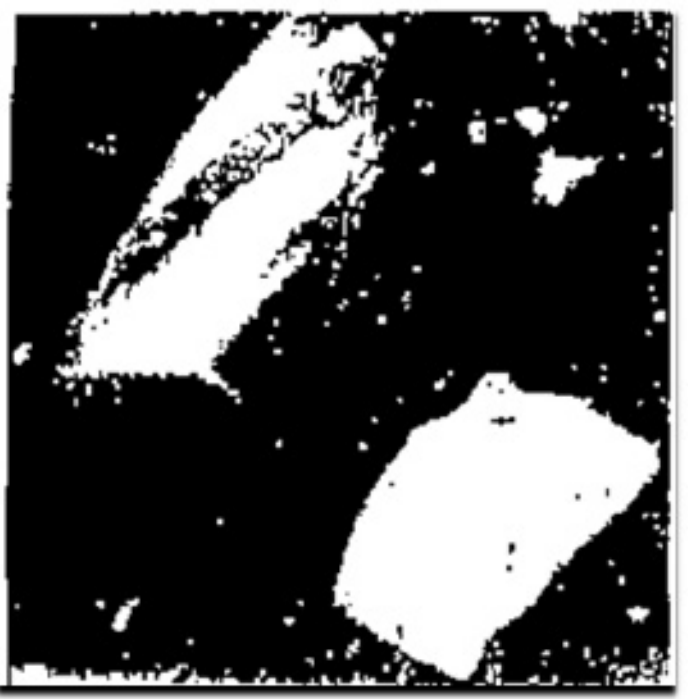

$75 \mu \mathrm{m}$

Figure 8. (a) Electron fractograph of composite specimens with $\mathrm{U}_{f}=0.05$ where plastic or viscous microflow conical- and candle-like markings of the matrix material around grains can be distinguished, and also, a nonuniformity in the grain size, and shape can be observed, b) Electron fractograph of composite specimen with $\mathrm{U}_{f}=0.10$ where plastic or viscous microflow conical and candle like markings around grains can be distinguished and also, the enhancement of the general microflow pattern in the matrix can be seen in comparison with Figure 8a. (c) Electron fractograph of composite specimen with $\mathrm{U}_{f}=0.25$ where a nonuniformity in the grain distribution, grain size and shape across the surface and also an enhancement of the general microflow patternand total debonding of grains from the matrix in comparison with Figure $8 \mathrm{~b}$ can be observed, (d) Same specimen as in Figure 8a-8c but taken at greater magnification in which some features concerning the microflow pattern such as microflow lines and decohesion gaps around and between the grains can easily be observed and where the nonuniformity in grain size and shape with many crevices can also be distinguished. 
This observed departure from the ideal linear elastic behavior (see Appendix B) may be caused, at least in part, to linear and nonlinear viscoelastic and plastic effects of the matrix. Moreover, because of the previously mentioned high stress- and strain concentrations, which exist locally between filler particles, the elastic limit of the matrix material may be exceeded long before the gross behavior of the composite exhibits the observed nonlinear response. The above statements are supported by the indicative fractograph (Fig. 8d) in which plastic or viscous microflow markings causing decohesion "gaps" between the matrix and grains were observed. In this sense it can be argued that the observed nonlinear response of the curve $\left(\mathrm{U}_{f}=0.20\right)$ in fact may be attributed mainly to such local viscoplastic or viscous flow, decohesion and total debonding effects.

Furthermore, the observed tendency of increase in the "radius of curvature" of the nonlinear range with increasing volume fraction of the plots given in Figure 3 may attributed mainly to the coupling effects between the above energy dissipative processes of the matrix and to the increase in the nonuniformity of the distribution density of the grains in the matrix (compare, for example, Fig. 8a with Fig. $8 \mathrm{~b}$ and $8 \mathrm{c}$ ).

In closure, it can be deduced from the foregoing text that the observed tendency of an "asymptotically stabilizing" behavior concerning the relative toughness in function of volume fraction should be, in the greatest part, attributed to an enhanced contribution of local viscoelastic-plastic failure energy absorbing effects in the reinforced matrix.

\section{Conclusions}

- In this article a theoretical model for the calculation of the elastic modulus of particulate composites was developed. In this development the influence of neighboring inclusions on the stiffness of the composite was examined by transforming a microstructural basic cell to a three-phase representative volume element. The theoretical values derived by the proposed model were compared with those given by other scientists and with experimental values obtained from tensile tests performed on composite material consisting of epoxy resin matrix and iron particles in various filler contents. It was observed that the theoretical values derived by the variations (M1) and (M3) of the proposed model presented large discrepancy with the experimental results since they were always too far. Only the variation (M2) of the present model yields values that are in agreement with experimental results. This conclusion proves that the distribution of the inclusion affects the elastic modulus of the composite.

- By means of a simple semiquantitative approach and fractographic data it can be shown that the discrepancies observed between the theoretical predictions and experimental data concerning the elastic modulus can be, in a great part, attributed to the inhomogeneity in the grain distribution in the matrix and to the variations of the finite interphase thickness due to the appreciable stress-strain concentration fields arising around the nonuniform shaped grains and causing local viscoelastic-plastic deformations of the epoxy matrix.

- In contrast to the reduced fracture stress and strains, which show a monotonic decreasing behavior by increasing the filler volume fraction, the related toughness of the composite shows an asymptotically stabilizing tendency. By the aid of fractographical data this behavior can be explained by the increased contribution of the local viscoplastic energy absorbing effects or total debonding, which tend to compensate the loss in fracture energy resulting from the apparent decrease in the fracture stress and strain.

\section{Appendix A: Theoretical Expressions of the Literature for $E_{c}$ and $\sigma_{c}$}

\section{Elastic Modulus}

Several number of theoretical or semiempirical equations for the effective moduli exist in the literature. Some of them are based on the theory of elasticity, some others use a mechanics of material approach or express a kind of law of mixtures, and still others try to match theoretical expressions to experimental values by appropriately defining the existing constants in these expressions.

\section{Einstein's Formula}

One of the theories concerning inclusions in a viscous matrix was developed by Einstein [38]. He considered rigid spherical non isolated particles in a Newtonian viscous fluid and expressed the viscosity in the form

$$
E_{c}=E_{\mathrm{m}}\left(1+2.5 U_{\mathrm{f}}\right)
$$

Einstein has also dealt with the theory of internal friction of spheres in a fluid that does not exert cohesion forces on them. In this case

$$
E_{c}=E_{m}\left(1+U_{f}\right)
$$

\section{Guth-Smallwood's Formula}

According to a formula developed by Guth and Smallwood [35, 36], which is an extension of Einstein's formula, the elastic modulus is given as

$$
E_{c}=E_{\mathrm{m}}\left(1+2.5 U_{\mathrm{f}}+14.1 \mathrm{U}_{\mathrm{f}}^{2}\right)
$$




\section{Counto's Formula}

The elastic modulus of a particulate composite according to Counto [40] is given as

$$
\overline{E_{c}}=\frac{-U_{\mathrm{f}}^{1 / 2}}{E_{m}}+\frac{}{\left(1-U_{\mathrm{f}}^{1 / 2}\right) U_{\mathrm{f}}^{1 / 2} E_{m}+E_{\mathrm{f}}}
$$

\section{Paul's Formula}

According to Paul [37], the elastic modulus is given as

$$
E_{c}=E_{m} \frac{+(m-1) U_{\mathrm{f}}^{2 / s}}{+(m-1)\left(U_{\mathrm{f}}^{2 / s}-U_{\mathrm{f}}\right)}
$$

where

$$
m=\frac{E_{\mathrm{f}}}{E_{m}}
$$

\section{Kerner's Formula}

A formula based on a mathematical model valid for the particulate composites was developed by Kerner [2].

$$
\frac{E_{c}}{E_{m}}=\frac{\frac{U_{f} G_{f}}{\left(7-5 v_{m}\right) G_{m}+\left(8-10 v_{m}\right) G_{\mathrm{f}}{ }^{+} 15\left(1-v_{m}\right)}}{\frac{U_{\mathrm{f}} G_{m}}{\left(7-5 v_{m}\right) G_{m}+\left(8-10 v_{m}\right) G_{\mathrm{f}}{ }^{+}{ }^{15\left(1-v_{m}\right)}}}
$$

where $G$ is the shear modulus of the composite for particles stiffer than matrix. Equation $A 6$ can be simplified as follows:

$$
\frac{E_{c}}{E_{m}}=1+\frac{U_{\mathrm{f}}}{U_{m}} \frac{15\left(1-v_{m}\right)}{\left(8-10 v_{m}\right)}
$$

\section{Takahashi's Formula}

Takahashi [39] derived the following expression for the elastic modulus.

$$
\frac{E}{E}=1+(1-v) U_{f} \frac{E_{f}(1-2 v)-E\left(1-v_{f}\right)+10(1+v) E_{f}(1+v)-E(1+v)}{E_{f}(1+v)+2 E\left(1-2 v_{f}\right)+2 E_{f}(4-5 v)(1+v)+E(7-5 v)\left(1+v_{f}\right)}
$$

\section{Ultimate stress}

Nielsen [31]

$$
\sigma_{t I}=\sigma_{m u}\left(1-U_{f}^{-2 / 3}\right) K
$$

where $\sigma_{c u}=\sigma_{m u}$ denote ultimate stress of composite and matrix respectively and $\mathrm{K}=0.5$

Nicolais [32]

$$
\sigma_{c u}=\sigma_{m i s}\left(1-1.21 U_{f}^{2 / 3}\right)
$$

Schrager [33]

$$
\sigma_{c L}=\sigma_{m u} \exp \left(-r v_{j}\right)
$$

where the constant equals to 2.66

\section{Appendix B: About Local (Intermolecular) Stress-Strain Relationships in Perfect Elastic Solid}

The potential energy function of a pair of atoms (molecules) is given by Joffe's general equation:

$$
\mathrm{U}=-\mathrm{A} / r^{\mathrm{m}}+\mathrm{B} / r^{\mathrm{m}}
$$

where $\mathrm{A}$ and $\mathrm{B}$ are constants; $m$ and $n$ are the exponents of the energy due only to attractive or repulsive forces respectively with $n>m$ always and $r$ may be an equivalent or effective intermolecular spacing.

The minimum of the potential energy, i.e. the bond energy $U=U_{0}, \quad$ is obtained at $r=r_{0}$, when $d U / d r=0$ and $A m / r_{0}{ }^{\mathrm{m}+1}=\mathrm{Bn} / \mathrm{r}_{0}{ }^{\mathrm{n}+1}$. Noting this relationship in $E q . B 1$ we obtain for the bonding forces an expression similar to that proposed by A.H. Cottrell:

$$
\frac{d U}{d r}=P=\frac{A m}{r_{o}} m+1\left[\left(\frac{r_{0}}{r}\right)^{m+1}-\left(\frac{r_{0}}{r}\right)^{n+1}\right]
$$

From this expression it follows that if a solid is subjected to mechanical loading such as tension, compression, or shear, the equilibrium interatomic (intermolecular) spacing $r_{0}$ may change to $r=r_{0} \pm \Delta r$. The greatest elastic interatomic or intermolecular displacement will be described by the relationship

$$
\frac{d P}{d r}=0, \text { or } r_{\max }=r_{0}\left(\frac{n+1}{m+1}\right)^{1 /(n-m)}
$$

As mentioned in the text, the changes in the interphase thickness, $\Delta r$ involve intermolecular displacements that are sustained mainly by changes in the van der Waal's bonding forces, for which $n=11$ and $m=6$. For this case from $E q$. $B 3$ we obtain that $+\Delta r_{\max }=0.115 r_{0}$ for tension. For compression changes $(-\Delta r)$ the maximal value can be greater. Now, from $E q$. $B 2$ we obtain the corresponding stress, $\sigma$.

$$
\sigma=\frac{A_{1} n}{r_{0}^{m+1}}\left[(1+\varepsilon)^{-(m+1)}-(1+\varepsilon)^{-(n+1)}\right]
$$

where $A_{1}$ is a constant proportional to $A$ and $\varepsilon=\Delta \mathrm{r} / \mathrm{r}_{0}$ is characteristic of strain in Lagrangian notation.

By expanding the term in brackets in $E q$. $B 4$ we obtain

$$
\begin{aligned}
\sigma & =\frac{A_{1} m(n-m)}{r_{0}+1} \times\left[\varepsilon+\frac{(m+1)(m+2)-(n+1)(n+2)}{(n-m) 2 !} \varepsilon^{2}\right. \\
& \left.+\frac{(m+1)(m+2)(m+3)-(n+1)(n+2)(n+3)}{(n-m) 3 !} \varepsilon^{3}+\cdots\right]
\end{aligned}
$$


Denoting the constant for a given solid, $\frac{\boldsymbol{A}_{1} m(n-m)}{r_{0}^{m+1}}$, as the local modulus of elasticity $\tilde{E}$ we find that the stressstrain relationship for ideal (perfect) solids is nonlinear and that the deviations from Hooke's law $\sigma=\tilde{E} \cdot \varepsilon$, i.e. the first term in $E q . B 5$, can be insignificant only for (very) low deformations or low strains. In the following table some of such deviations are given for certain applied strains.

\begin{tabular}{lc|}
\hline Applied strain (\%) & Deviation from Hooke's Law \\
\hline 1 & 12 \\
2 & 28 \\
3 & 40 \\
4 & 56 \\
\hline
\end{tabular}

Because of the fact that some epoxy resins (or other "brittle" polymers) although nonperfect (real) solid, may possess relatively high elastic limit, about $4 \%$, from this table the possibility of an appreciable contribution of a nonlinear component to the linear elastic behavior becomes clear. (This time independent nonlinear elastic behavior may be expressed in the general viscoelastic modeling by nonlinear Hookean springs.) Furthermore, from the above introduced expression,

$$
\tilde{E}(r)=\frac{A_{1} m(n-m)}{r^{m+1}} \text {, we obtain the differential }
$$

$$
\Delta \tilde{E}(r)=\frac{\partial \tilde{E}}{\partial r} \Delta r
$$

which gives

$$
\Delta \tilde{E}(r)=\frac{-A_{1} m(n-m)(m+1)}{r^{m+2}} \Delta r
$$

For $\mathrm{m}=6, \mathrm{n}=11, \mathrm{r}=$ ro and in view of simplicity one may assume that $|+\Delta r|_{\max }=|-\Delta r|_{\max } \cong 0.115 r_{0}$ and thus we can estimate the ratio $(\Delta \tilde{E}) / \tilde{E}$ as follows

$$
\frac{\Delta \tilde{E}}{\tilde{E}}=\frac{-(m+1)}{r_{0}}( \pm \Delta r) \cong 0.80
$$

This means that possible changes in the local modulus of elasticity $\tilde{E}$ (around the grain) can reach values as high as \pm 0.80 for changes $( \pm \Delta r) \cong 0.115$ in the interphase thickness between grain filler and polymeric matrix

\section{REFERENCES}

[1] L.E. Nielsen, Mechanical Properties of Polymers and Composites, Vol. 2, Marcel Dekker, New York (1974).

[2] E.H. Kerner, Proc. Phys. Soc., 69B, 808 (1956).

[3] Z. Hashin and S. Shtrikman, J. Mech. Phys. Solids, 11, 127
(1963).

[4] Z. Hojo, W. Toyoshima, M. Amura, and N. Kawamura, Polym. Eng. Sci., 14, 604 (1974).

[5] H. Alter, J. Appl. Polym. Sci., 9, 1525 (1966).

[6] W.M. Baldin, Acta Mech., 6, 141 (1958).

[7] R.M. Christensen and K.H. Lo, J. Mech. Phys. Solids, 27, 315 (1979).

[8] R.M. Christensen, J. Mech. Phys. Solids, 38, 379 (1990).

[9] T.S. Chow,Polym. Sci., 16, 959 (1978).

[10] P.S. Theocaris, J. Reinforc. Plast. Compos., 3, 204 (1984).

[11] P.S. Theocaris, J. Appl. Polym. Sci., 30, 621 (1985).

[12] S.K. Bhatacharya, S. Basu, and S.K. De, J. Mater. Sci., 13, 2109 (1978).

[13] G. Landon, G. Lewis, and G.F. Boden, J. Mater. Sci., 12,1605 (1977).

[14] A. Malliaris and D.T. Turner, J. Appl. Phys., 42, 614 (1971).

[15] G.W. Brassell and K.B. Wischmann, SPE J., 9, 307 (1974).

[16] E. Sideridis, P.S. Theocaris, and G.C. Papanicolaou, Rheol. Acta., 25, 350 (1986).

[17] E. Sideridis and G.C. Papanicolaou, Rheol. Acta., 27, 608 (1988).

[18] F.H. Maurer, R. Simha, and R.K. Jain, "On the Elastic Moduli of Particular Composites: Interlayer versus Molecular Model," in Composite Interfaces, H. Ishida et al., eds., North-Holland, 367 (1986).

[19] Y. Benveniste, Mech. Mater., 4, 197 (1985).

[20] J. Aboudi, Compos. Sci.Technol., 28, 103 (1987).

[21] Z. Hashin, Mech. Mater., 8, 333 (1990).

[22] Sideridis E., Venetis J., Thermal expansion coefficient of particulate composites defined by the particle contiguity, Int. J. of Micr. Mat.Properties, 9, 292-313, (2014)

[23] J. Venetis and E. Sideridis, Thermal conductivity coefficient of particulate composites as defined by the particle arrangement, International Journal of Applied Engineering Research 10(14), 34230-34237, (2015).

[24] D.K. Balch, T.J. Fitzgerald, V.J. Michaud, A. Mortensen, Y.-L. Shen, S. Suresh Thermal Expansion of Metals Reinforced with Ceramic Particles and Microcellular Foams Metallurgical and Materials Transactions A Volume 27, Issue 11, 3700-3717, (1996)

[25] G. Roudini, R. Tavangar, L. Weber, A. Mortensen: Influence of reinforcement contiguity on the thermal expansion of alumina particle reinforced aluminium composites, Int. J. Mat. Res. 101, 1113-1120 (2010)

[26] J. Venetis, E. Sideridis, A mathematical model for thermal conductivity of homogeneous composite materials Indian Journal of Pure \& Applied Physics Vol. 54, 313-320 (2016)

[27] S. J. Torquato, Effective stiffness tensor of composite 
media. II. Applications to isotropic dispersions, J. Mech Phys. Solids 46(8) ,1411(1998)

[28] L. V. Gibianski and S. J. Torquato, New method to generate free-point bounds on effective properties of composites: application to viscoelasticity, J. Mech. Phys.Solids 46(4), 749-783, (1998).

[29] J. P. O'Rourke, M. S. Ingber and M. W. Weiser, The effective elastic constants of solids containing spherical exclusions, J. Compos. Mater. 31(9), 910-934 (1997)

[30] H. M. Yin and L. Z. Sun, Elastic modeling of periodic composites with particle interactions, Philos. Mag. Lett. 85(4) , 163-173 (2005)

[31] Nielsen, L. E.: Mechanical Properties of Particulate-Filled Systems, J. Compo Mat. 1,pp. 100-119 (1967)

[32] L. Nicolais, Polym. Eng. Sci., 11, 194 (1971)

[33] Schrager M (1978) The effect of spherical inclusions on the ultimate strength of polymer composites. J Appl Polym Sci 22:2379-2381.

[34] Y.S. Lipatov, Physical Chemistry of Filled Polymers (Translated from Russian by R.J. Moseley), Int. Polym. Sci. Technol. Monograph No. 2 (1977).

[35] E. Guth, J. Appl. Phys., 16, 20 (1945).
[36] H.M. Smallwood, J. Appl. Phys., 15, 758 (1944).

[37] B. Paul, Trans. Am. Inst. Mech. Eng., 218, 1017, (1960).

[38] A. Einstein, Uber die von molekularkinetischen Theorie der Warme Geforderto Bewegung von in Ruhenden Flussigkeiten suspendionten Teilchen, Ann. Physik, 17, 549 (1905); (b) Eine Neue bestimmung derMolekuldimensione n, Ann. Physik., 19, 289 (1906); (c) Berichtigung zu Meiner Arbeit: Eine Neue Bestimmung der Molekuldimensionen, 34, $591(1911)$

[39] K. Takahashi, M. Ikeda, K. Harakawa, and K. Tanaka, $J$ Pol. Pjys., Ed., 16, 415 (1978).

[40] R. Counto, Mag. Concr. Res., 16, 129 (1964).

[41] P.S. Theocaris, The Mesophase Concept in Composites, Springer-Verlag, Berlin (1987).

[42] J. Beny, in Fracture Processes in Polymeric Solids-Phenomena and Theory, B. Rosen, ed., Wiley, New York (1964).

[43] P. Polukhin, S. Gorelik, and V. Vorontsov, Physical Principles of Plastic Deformation, Mir Publishers, Moscow (1983) 